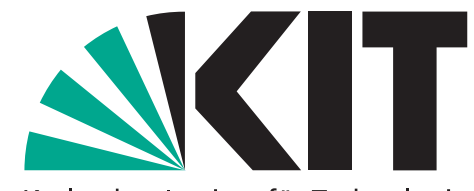

Karlsruher Institut für Technologie

\title{
Uniqueness of Markov equilibrium in stochastic OLG models with nonclassical production
}

by Marten Hillebrand

No. 46 | NOVEMBER 2012

\section{WORKING PAPER SERIES IN ECONOMICS}

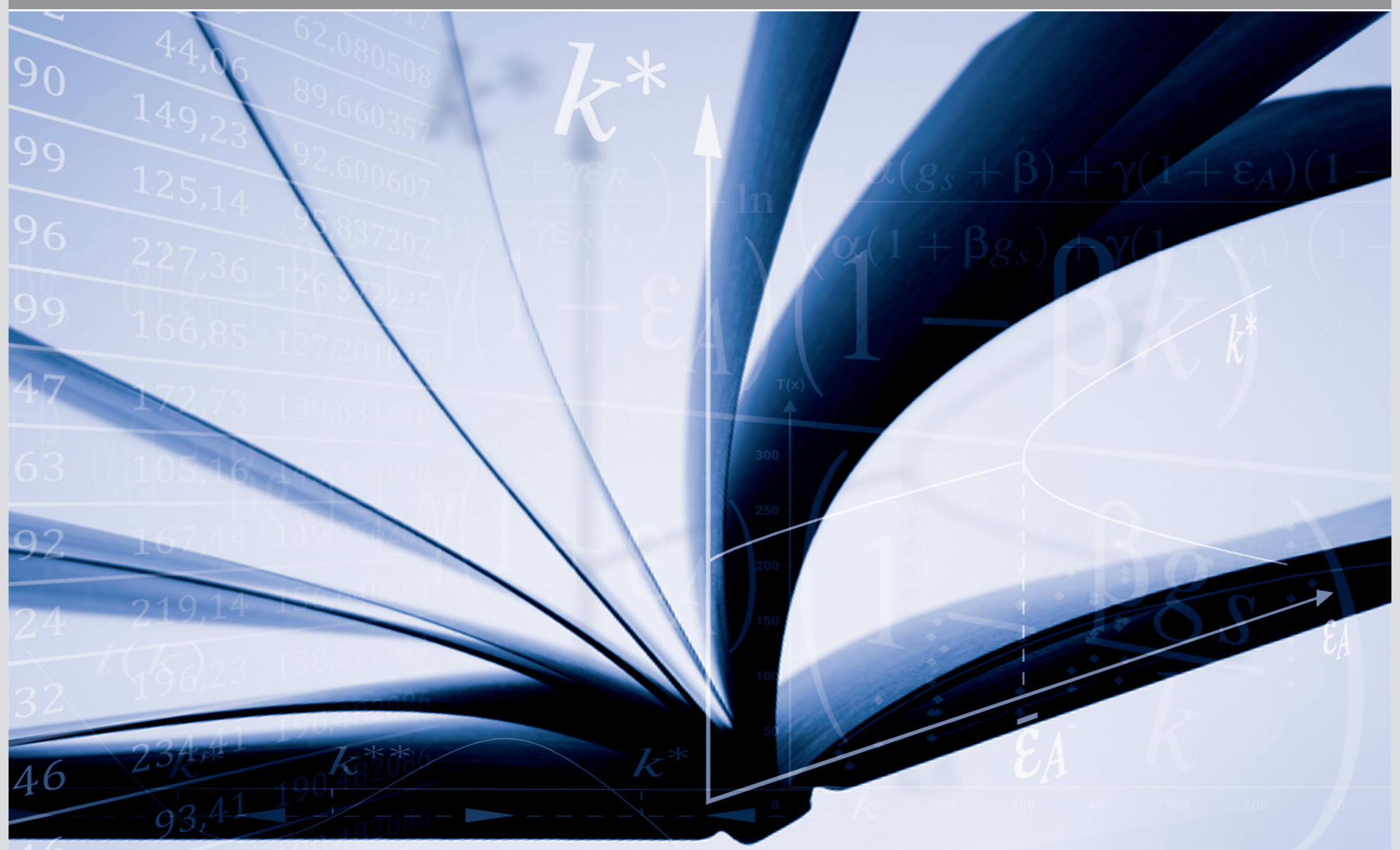




\section{Impressum}

Karlsruher Institut für Technologie (KIT)

Fakultät für Wirtschaftswissenschaften

Institut für Wirtschaftspolitik und Wirtschaftsforschung (IWW)

Institut für Wirtschaftstheorie und Statistik (ETS)

Schlossbezirk 12

76131 Karlsruhe

KIT - Universität des Landes Baden-Württemberg und nationales Forschungszentrum in der Helmholtz-Gemeinschaft

Working Paper Series in Economics

No. 46, November 2012

ISSN 2190-9806

econpapers.wiwi.kit.edu 


\title{
Uniqueness of Markov Equilibrium in Stochastic OLG Models with Nonclassical Production*
}

\author{
Marten Hillebrand ${ }^{\dagger}$
}

November 27, 2012

\begin{abstract}
This paper studies Markov Equilibria (ME) corresponding to recursive equilibria on the natural state space in the stochastic OLG model extended to include non-additive utility, nonclassical production, and Markovian production shocks. Specifically, we provide sufficient conditions under which the ME is unique. It turns out that uniqueness obtains for a large class of economies and that restrictions either on the consumption side or the production side alone are sufficient to guarantee this result. We also discuss additional properties such as continuity or smoothness of the equilibrium mappings and whether additional recursive or non-recursive equilibria exist.
\end{abstract}

JEL classification: C62, D51, E32.

Keywords: Markov equilibrium, Uniqueness, Overlapping generations, Nonclassical production, Markovian production shocks

*I would like to thank Martin Barbie, Tomoo Kikuchi, Caren Söhner, and John Stachurski for helpful discussions and comments.

${ }^{\dagger}$ Karlsruhe Institute of Technology, Department of Economics, Germany, Email: marten.hillebrand@kit.edu, Tel.: +49721608 45667 


\section{Introduction}

Starting with the pioneering work of Wang (1993), researchers have studied the existence and properties of equilibria in overlapping generations (OLG) models with random production shocks. Of particular interest in these studies is the class of recursive equilibria (RE) where the equilibrium variables are determined by time-invariant mappings on the natural state space with the state variable consisting of current capital and the production shock. Following the terminology introduced in Kübler \& Polemarchakis (2004), such equilibria will be referred to as Markov equilibria (ME). Studying the properties of ME for a large class of stochastic OLG models is the theme of the present paper.

In a setting with classical production functions, i.i.d. shocks, and time-additive utility, Wang (1993) showed that a capital-income monotonicity condition on the production technology is sufficient for a unique ME to exist. In addition, he established several additional properties of the equilibrium mappings such as smoothness and monotonicity. The model in Wang (1993) was generalized in Wang (1994) to include non-additive utility and general Markovian shocks processes and further in Morand \& Reffett (2007) who, in addition, allow for non-classical production functions. The latter were originally introduced in Greenwood \& Huffman (1995) and Coleman (1991) in a non-OLG setting. While Wang (1994) uses the methods and results of Duffie et a. (1994) to study the existence of so-called Generalized Markov Equilibria (GME) where the state space may include additional variables such as sunspots, etc., Morand \& Reffett (2007) continue to focus on ME and present a very general approach to study their existence. Their approach employs methods from functional analysis to obtain ME as fixed points of a suitably defined operator. Using the Knaster-Tarski fixed point theorem permitted them to derive sufficient conditions for a ME to exist in their setup.

Building on the existence results of Morand \& Reffett (2007), the present paper seeks to establish additional properties of ME while maintaining the same level of generality as their study. As our main contribution, we establish sufficient conditions under which the ME is unique and the equilibrium mappings possess additional properties such as monotonicity, continuity, or even smoothness. Similar properties were derived in Wang (1993) but it is not yet known under what conditions they hold for the much larger class of economies studied in Morand \& Reffett (2007). Knowing these additional properties is important not only for theoretical reasons and welfare analysis, but also for applied numerical work as, e.g., in Feng et al. (2012).

Conceptually, we employ the same operator-based approach used in Morand \& Reffett (2007). In general, uniqueness of a fixed point obtains only under very special circumstances, e.g., if the underlying operator is a contraction or satisfies a set of additional and rather restrictive conditions as in Coleman (1991). However, we demonstrate in this paper that the operator developed in Morand \& Reffett (2007) possesses a very special structure that is unique to their OLG setup with two-period lived consumers 
but not exploited in their paper. It is precisely this additional property that will allow us to obtain the uniqueness results of this paper. In fact, it will turn out that the ME is unique for most cases studied in Morand \& Reffett (2007). Moreover, we also show that if the ME is unique, it is in fact the unique sequential equilibrium of the economy.

The paper is organized as follows. Section 1 introduces the model. The formal structure to study ME is developed in Section 2. The main results are presented in Section 3. Section 4 concludes, proofs for all results are placed in the Mathematical Appendix.

\section{The Model}

This section presents the basic setup of the model which extends the one in Morand \& Reffett (2007) by relaxing several of their assumptions.

\section{Consumption sector}

The consumption sector consist of overlapping generations of consumers who live for two periods. In their first period of life, consumers supply one unit of labor inelastically to receive the wage $w_{t}>0$ which is used for saving $s_{t}$ and consumption when young $c_{t}^{y}=$ $w_{t}-s_{t}$. Savings earn the random capital return $r_{t+1}$ in the following period in which no further income is received such that second-period consumption is given by the random variable $c_{t+1}^{o}=s_{t} r_{t+1}$. Given labor income $w_{t}>0$ and the perceived random capital return of the following period $r_{t+1}$, consumers choose savings $s_{t}$ to maximize expected lifetime utility based on some von-Neumann Morgenstern utility function function $U$ : $\mathbb{R}_{+}^{2} \longrightarrow \mathbb{R}$ defined over consumption in both periods. The decision problem reads

$$
\max _{s}\left\{\mathbb{E}_{t}\left[U\left(w_{t}-s, s r_{t+1}\right)\right] \mid 0 \leq s \leq w_{t}\right\}
$$

Here, $\mathbb{E}_{t}$ denotes the expectations operator conditional on information at time $t$ which is formally defined below. The following restrictions are imposed on $U$.

\section{Assumption 1}

$U$ is $C^{2}$ and concave with derivatives satisfying $U_{i i}<0<U_{i}, i \in\{1,2\}$ and the Inada conditions $\lim _{c^{y} \rightarrow 0} U_{1}\left(c^{y}, c^{o}\right)=\infty$ for all $c^{o}>0$ and $\lim _{c^{o} \rightarrow 0} U_{2}\left(c^{y}, c^{o}\right)=\infty$ for all $c^{y}>0$.

Given $w_{t}>0$ and the random variable $r_{t+1}$, Assumption 1 guarantees a unique interior solution $0<s_{t}<w_{t}$ to (1) which determines next period's capital stock $k_{t+1}$.

Production sector

The production sector produces the consumption good using labor and capital as input factors. In addition, the production process in period $t$ is subjected to a random shock $\varepsilon_{t}$ with values in $\mathcal{E} \subset\left[\varepsilon_{\min }, \varepsilon_{\max }\right]$. In equilibrium, labor is constant and the wage $w_{t}$ and capital return $r_{t}$ are determined from the current stock of capital $k_{t}>0$ and the shock 
$\varepsilon_{t} \in \mathcal{E}$ according to the mappings

$$
\begin{aligned}
\mathcal{W}: \mathbb{R}_{++} \times \mathcal{E} \longrightarrow \mathbb{R}_{++}, & w_{t}=\mathcal{W}\left(k_{t}, \varepsilon_{t}\right) \\
\mathcal{R}: \mathbb{R}_{++} \times \mathcal{E} \longrightarrow \mathbb{R}_{++}, & r_{t}=\mathcal{R}\left(k_{t}, \varepsilon_{t}\right) .
\end{aligned}
$$

Profits are zero at equilibrium. The previous specification includes the cases with classical production in Wang (1993) and non-classical production in Greenwood \& Huffman (1995), Coleman (1991), and Morand \& Reffett (2007) as special cases. Rather than specifying the underlying production technology directly, we will work with the mappings $\mathcal{W}$ and $\mathcal{R}$ as being part of the primitives of the economy. The following restrictions are imposed which are slightly weaker than those in Morand \& Reffett (2007).

\section{Assumption 2}

(i) Both functions $\mathcal{W}$ and $\mathcal{R}$ are Borel-measurable.

(ii) For each $\varepsilon \in \mathcal{E}, k \mapsto \mathcal{W}(k, \varepsilon)$ is increasing while $k \mapsto \mathcal{R}(k, \varepsilon)$ is strictly decreasing.

(iii) For each $k>0, \varepsilon \mapsto \mathcal{W}(k, \varepsilon)$ and $\varepsilon \mapsto \mathcal{W}(k, \varepsilon)$ are bounded.

Production shocks follow a Markov process with time-invariant transition probability $Q: \mathcal{E} \times \mathscr{B}(\mathcal{E}) \longrightarrow[0,1]$. Given an initial state $\varepsilon_{0} \in \mathcal{E}$, the transition $Q$ permits to construct a probability space $(\Omega, \mathcal{F}, \mathbb{P})$ together with a filtration $\left\{\mathcal{F}_{t}\right\}_{t>0}$ to which the process $\left\{\varepsilon_{t}\right\}_{t \geq 0}$ is adapted such that $\varepsilon_{t}: \Omega \longrightarrow \mathcal{E}$ is $\mathcal{F}_{t}$-measurable, $t \geq 0$. ${ }^{1}$ We denote by $\mathbb{E}_{t}[\cdot]:=\mathbb{E}\left[\cdot \mid \mathcal{F}_{t}\right]$ the expectation conditional on the information represented by $\mathcal{F}_{t}$. The following assumption adds some technical restrictions on $Q$.

\section{Assumption 3}

$Q$ preserves measurability, i.e., if $f: \mathbb{Y} \times \mathcal{E} \longrightarrow \mathbb{R}, \mathbb{Y} \subset \mathbb{R}^{m}, m \geq 1$ is a measurable function, then $g: \mathbb{Y} \times \mathcal{E} \longrightarrow \mathbb{R}, g(y, \varepsilon):=\int_{\mathcal{E}} f\left(y, \varepsilon^{\prime}\right) Q\left(\varepsilon, d \varepsilon^{\prime}\right)$ is also measurable.

A stronger requirement frequently imposed would be the so-called Feller property: If $\mathbb{Y} \subset \mathbb{R}^{m}, m \geq 1$ and $f: \mathbb{Y} \times \mathcal{E} \longrightarrow \mathbb{R}$ is a bounded continuous function, then so is $g: \mathbb{Y} \times \mathcal{E} \longrightarrow \mathbb{R}, g(y, \varepsilon):=\int_{\mathcal{E}} f\left(y, \varepsilon^{\prime}\right) Q\left(\varepsilon, d \varepsilon^{\prime}\right)$. We will refrain from imposing this property directly but indicate below how it would permit to sharpen some results.

\section{Equilibrium}

The economy is $\mathscr{E}=(U, \mathcal{W}, \mathcal{R}, Q)$ plus the initial condition $x_{0}:=\left(k_{0}, \varepsilon_{0}\right) \in \mathbb{X}:=$ $\mathbb{R}_{++} \times \mathcal{E}$. The set $\mathbb{X}$ will be referred to as the (natural) state space of the economy. The following definition provides a general characterization of equilibrium. Note that the previous assumptions imply that all equilibrium variables are strictly positive.

\footnotetext{
${ }^{1}$ In what follows, the notion of an adapted stochastic process $\left\{\xi_{t}\right\}_{t \geq 0}$ taking values in a topological space $\Xi$ implies that each $\xi_{t}: \Omega \longrightarrow \Xi$ is $\mathcal{F}_{t}$ measurable, i.e., can depend only on the random variables $\left(\varepsilon_{0}, \ldots, \varepsilon_{t}\right)$. Measurability of mappings $M: \mathbb{Y} \longrightarrow \mathbb{Z}$ between topological spaces $\mathbb{Y}$ and $\mathbb{Z}$ is always understood with respect to the Borel $\sigma$-algebras $\mathscr{B}(\mathbb{Y})$ and $\mathscr{B}(\mathbb{Z})$.
} 


\section{Definition 1}

Given $x_{0} \in \mathbb{X}$, a sequential equilibrium (SE) of economy $\mathscr{E}$ is an adapted process $\left\{w_{t}, r_{t}, s_{t}, k_{t+1}\right\}_{t \geq 0}$ with values in $\mathbb{R}_{++}^{4}$ satisfying the following conditions for all $t \geq 0$ :

(i) Given $w_{t}$ and the random variable $r_{t+1}, s_{t}$ solves (1) while $k_{t+1}=s_{t}$.

(ii) Factor prices $w_{t}$ and $r_{t}$ are determined from $k_{t}$ and $\varepsilon_{t}$ by (2a) and (2b).

The induced equilibrium consumption processes $\left\{c_{t}^{y}, c_{t}^{o}\right\}_{t \geq 0}$ follow directly by inserting the equilibrium variables into the consumers' budget constraints.

\section{Markov Equilibria (ME)}

\section{Definition of $M E$}

A recursive equilibrium (RE) is an equilibrium where all equilibrium variables of period $t$ are determined by time-invariant functions of some state variable $x_{t}$ taking values in the state space $\mathbb{X}$. A Markov equilibrium (ME) is a recursive equilibrium on the natural state space $\mathbb{X}=\mathbb{R}_{++} \times \mathcal{E}$ where the state variable is $x_{t}:=\left(k_{t}, \varepsilon_{t}\right)$. It is this class of equilibria that we will focus on in this paper. As the functions $\mathcal{W}$ and $\mathcal{R}$ already satisfy the Markov property, a ME is essentially determined by a time-invariant mapping which determines the evolution of capital respectively savings. Formally, we have

\section{Definition 2}

Given $x_{0} \in \mathbb{X}$, a $M E$ of economy $\mathscr{E}$ is a measurable map $\mathcal{K}: \mathbb{X} \longrightarrow \mathbb{R}_{++}$on the natural state space $\mathbb{X}=\mathbb{R}_{++} \times \mathcal{E}$ such that the process $\left\{w_{t}, r_{t}, s_{t}, k_{t+1}\right\}_{t \geq 0}$ defined recursively as $k_{t+1}=\mathcal{K}\left(k_{t}, \varepsilon_{t}\right)=s_{t}, w_{t}=\mathcal{W}\left(k_{t}, \varepsilon_{t}\right)$, and $r_{t}=\mathcal{R}\left(k_{t}, \varepsilon_{t}\right)$ for all $t \geq 0$ is a SE of $\mathscr{E}$.

\section{Constructing the operator A}

To establish the existence and properties of ME, we follow Morand \& Reffett (2007) to construct an operator $A$ on a suitable function space $\mathscr{S}$ whose fixed points are ME. In the sequel we take $\mathscr{S}$ to be the class of Borel-measurable functions $\mathcal{K}: \mathbb{X} \longrightarrow \mathbb{R}_{++}$. The operator $A$ is constructed from the Euler equations derived from the consumer's decision problem (1). To this end, consider a given period $t$ with state $x_{t}=\left(k_{t}, \varepsilon_{t}\right)$ which determines the current wage $w_{t}$ from $(2 \mathrm{a})$ and the conditional distribution $Q\left(\varepsilon_{t}, \cdot\right)$ of next period's shock. To decide on her investment $s_{t} \in\left[0, w_{t}\right]$, the consumer needs to determine the (correct) distribution of the uncertain capital return $r_{t+1}$ of the following period. As $r_{t+1}=\mathcal{R}\left(k_{t+1}, \varepsilon_{t+1}\right)$ and the consumer knows the function $\mathcal{R}$ and the conditional distribution of $\varepsilon_{t+1}$ (which are part of the fundamentals of the economy), this amounts to (correctly) forecasting next period's capital stock $k_{t+1}>0$ which, conditional on the information at time $t$, is a value rather than a random variable. Suppose the consumer holds a perceived law of motion for the capital stock $\mathcal{K} \in \mathscr{S}$ to compute her forecast 
$k_{t+1}=\mathcal{K}\left(k_{t}, \varepsilon_{t}\right)$. Then, given $\left(x_{t}, k_{t+1}\right) \in \mathbb{X} \times \mathbb{R}_{++}$, an optimal savings decision $s_{t}$ derived from (1) must satisfy the first-order conditions $H\left(s_{t} ; k_{t}, \varepsilon_{t}, k_{t+1}\right)=0$ where

$$
\begin{aligned}
H\left(s ; k, \varepsilon, k^{\prime}\right):= & -\int_{\mathcal{E}} U_{1}\left(\mathcal{W}(k, \varepsilon)-s, s \mathcal{R}\left(k^{\prime}, \varepsilon^{\prime}\right)\right) Q\left(\varepsilon, d \varepsilon^{\prime}\right) \\
& +\int_{\mathcal{E}} \mathcal{R}\left(k^{\prime}, \varepsilon^{\prime}\right) U_{2}\left(\mathcal{W}(k, \varepsilon)-s, s \mathcal{R}\left(k^{\prime}, \varepsilon^{\prime}\right)\right) Q\left(\varepsilon, d \varepsilon^{\prime}\right) .
\end{aligned}
$$

Under the Inada assumptions (which ensure existence) and the concavity of $U$ (which implies uniqueness), the function $H\left(\cdot ; x, k^{\prime}\right)$ has a unique zero for all $x \in \mathbb{X}$ and $k^{\prime}>0$. Thus, there exists a (savings) function $\mathcal{S}: \mathbb{X} \times \mathbb{R}_{++} \longrightarrow \mathbb{R}_{++}$which determines the unique solution $s_{t}=\mathcal{S}\left(k_{t}, \varepsilon_{t}, k_{t+1}\right)$ to (1). The following lemma is the key ingredient to define the operator $A$ below.

\section{Lemma 2.1}

Under Assumptions 1, 2, and 3, the mapping $\mathcal{S}: \mathbb{X} \times \mathbb{R}_{++} \longrightarrow \mathbb{R}_{++}$is measurable.

Substituting the perceived law of motion $\mathcal{K}$ into $\mathcal{S}$ one obtains an operator $A$ which associates with $\mathcal{K} \in \mathscr{S}$ the new function $A \mathcal{K}: \mathbb{X} \longrightarrow \mathbb{R}_{++}$defined as

$$
(A \mathcal{K})(x):=\mathcal{S}(x, \mathcal{K}(x)) \text { for } x \in \mathbb{X} .
$$

As $S$ is measurable by Lemma 2.1 and the composition of measurable functions is again measurable, it is evident that $A$ maps $\mathscr{S}$ into itself. ${ }^{2}$ As $k_{t+1}=s_{t}$ at equilibrium, a ME corresponds precisely to a fixed point of $A$.

Fixed points of $A$

Under a set of additional restrictions, Morand \& Reffett (2007) establish the existence of fixed points of $A$. Their argument is based on the Knaster-Tarski fixed point theorem for which they impose additional restrictions such as monotonicity on the set $\mathscr{S}$.

In this paper, we follow a different route which determines fixed points of $A$ pointwise as zeroes of a real-valued function. This approach is possible due to a key property of the operator $A$ which is evident from (4) but not exploited in Morand \& Reffett (2007): For each $x \in \mathbb{X}$, the value $(A \mathcal{K})(x)$ depends only on the value $\mathcal{K}(x)$ and not on the entire function $\mathcal{K}$. Formally, for any two functions $\mathcal{K}_{1}, \mathcal{K}_{2} \in \mathscr{S}, \mathcal{K}_{1}(x)=\mathcal{K}_{2}(x)$ implies $\left(A \mathcal{K}_{1}\right)(x)=\left(A \mathcal{K}_{2}\right)(x) .{ }^{3}$ The main implication of this property is that fixed-points of $A$ can be constructed point-wise, for each state $x \in \mathbb{X}$. This fact is stated formally in the following lemma the proof of which follows directly from (4) and the previous arguments.

\footnotetext{
${ }^{2}$ It is straightforward to show that $A$ maps $\mathscr{S}$ into the class $\mathscr{S}^{\prime}$ of measurable functions $\mathcal{K}: \mathbb{X} \longrightarrow$ $\mathbb{R}_{++}$which satisfy, in addition, $\mathcal{K}(x)<\mathcal{W}(x)$ for all $x \in \mathbb{X}$. Thus, fixed points of $A$ must necessarily be elements of $\mathscr{S}^{\prime}$. If $\lim _{k \rightarrow 0} \mathcal{W}(0, \varepsilon)=0$ for all $\varepsilon \in \mathcal{E}$, one also infers the boundary behavior $\lim _{k \rightarrow 0} \mathcal{K}(k, \varepsilon)=0$ for any $\mathcal{K} \in \mathscr{S}^{\prime}$.

${ }^{3}$ This very special structure is unique to overlapping generations models with two-period lived consumers. In most macroeconomic models with multiperiod or infinitely-lived consumers, the operator $A$ will vary with the entire function $\mathcal{K}$, i.e., the value $(A \mathcal{K})(w)$ depends on the entire function $\mathcal{K}$.
} 


\section{Lemma 2.2}

The map $\mathcal{K} \in \mathscr{S}$ is a fixed point of $A$, i.e., $(A \mathcal{K})(x)=\mathcal{K}(x)$ for all $x \in \mathbb{X}$, if and only if

$$
\mathcal{S}(x, \mathcal{K}(x))=\mathcal{K}(x) \forall x \in \mathbb{X}
$$

General uniqueness conditions

The result from Lemma 2.2 will permit us to establish many additional properties of ME not derived in Morand \& Reffett (2007). In particular, we will provide sufficient conditions under which the economy has a unique ME. To prepare these results, let $\Phi: \mathbb{X} \times \mathbb{R}_{++} \longrightarrow \mathbb{R}$

$$
\Phi\left(x, k^{\prime}\right):=k^{\prime}-\mathcal{S}\left(x, k^{\prime}\right)
$$

Note that Lemma 2.1 implies that $\Phi$ is measurable. Using Lemma 2.2, it follows that for each $x \in \mathbb{X}$, the value $k^{\star}=\mathcal{K}^{\star}(x)$ of any fixed point $\mathcal{K}^{\star} \in \mathscr{S}$ of $A$ must must be a zero of $\Phi(x, \cdot)$, i.e.,

$$
\Phi\left(x, k^{\star}\right)=0 .
$$

Equation (7) is precisely the condition employed in de la Croix \& Michel (2002) in a deterministic setting. It is also equivalent to - in fact, merely a restatement of - the self-confirming expectations approach in Wang (1993). Define the correspondence

$$
\Psi: \mathbb{X} \rightrightarrows \mathbb{R}_{++}, \quad \Psi(x):=\left\{k^{\prime} \in \mathbb{R}_{++} \mid \Phi\left(x, k^{\prime}\right)=0\right\}
$$

Then, determining a $\mathrm{ME}$ is equivalent to finding a measurable selection of $\Psi$, i.e., a measurable function $\mathcal{K}: \mathbb{X} \longrightarrow \mathbb{R}_{++}$such that $\mathcal{K}(x) \in \Psi(x)$ for all $x \in \mathbb{X}$. Clearly, a necessary condition for ME to exist is that $\Psi$ be non-empty valued. It is also clear that if $\Psi$ is single-valued, i.e., a function, then there can be at most one ME. In this latter case, the next result shows that $\Psi$ will automatically be measurable, i.e., a unique $\mathrm{ME}$ exists. On the other hand, if for each $x \in \mathbb{X}$ the map $\Phi(x, \cdot)$ has at most one zero, there can be at most one ME. Thus, a sufficient condition for uniqueness is that $\Phi(x, \cdot)$ be strictly monotonic for all $x \in \mathbb{X}$. The following final result of this section summarizes these insights which will be key for the uniqueness result derived in the next section.

\section{Lemma 2.3}

Let $\Phi: \mathbb{X} \times \mathbb{R}_{++} \longrightarrow \mathbb{R}$ defined in (6) be measurable. Then, the following holds:

(i) $\mathscr{E}$ has a unique $M E$, if and only if $\Phi(\tilde{x}, \cdot)$ has a unique zero $\tilde{k}>0$ for each $\tilde{x} \in \mathbb{X}$.

(ii) If $\Phi(\tilde{x}, \cdot)$ has at most one zero for each $\tilde{x} \in \mathbb{X}$, then $\mathscr{E}$ has at most one ME.

\section{Uniqueness of Equilibrium}

Uniqueness of $M E$

Exploiting the insights from the previous section, we are now in a position to establish a 
set of additional properties of ME. The first main result is the following theorem which lists sufficient conditions under which the economy has at most one ME.

\section{Theorem 1}

Let Assumptions 1, 2, and 3 be satisfied, Then, each of the following restrictions is sufficient for the economy $\mathscr{E}$ to have at most one ME:

(i) $U\left(c^{y}, c^{o}\right)=u\left(c^{y}\right)+v\left(c^{o}\right)$ where $v$ satisfies $\frac{v^{\prime \prime}(c) c}{v^{\prime}(c)} \geq-1$.

(ii) $U_{12} \geq 0$ and $k \mapsto k \mathcal{R}(k, \varepsilon), k>0$ is weakly increasing for all $\varepsilon \in \mathcal{E}$.

(iii) $\frac{c^{o} U_{22}\left(c^{y}, c^{o}\right)}{U_{2}\left(c^{y}, c^{o}\right)}>-1$ for all $\left(c^{y}, c^{o}\right) \gg 0$ and $k \mapsto \mathcal{R}(k, \varepsilon)$ is differentiable for all $\varepsilon \in \mathcal{E}$ where $\mathcal{R}_{1}<0$. In addition, either $U_{21} \leq 0$ or $\frac{\mathcal{R}_{1}(k, \varepsilon) k}{\mathcal{R}(k, \varepsilon)} \geq-4$ for all $k>0, \varepsilon \in \mathcal{E}$.

The hypotheses of Theorem 1 are satisfied for a broad class of economies. Condition (i) holds, e.g., if second-period utility displays constant relative risk aversion $0<\theta \leq 1$, i.e., $v(c)=\frac{\gamma}{1-\theta}\left(c^{1-\theta}-1\right), \gamma>0$ or under CES utility $v(c)=\left[1-\theta+\theta c^{\varrho}\right]^{1 / \varrho}, 0<\theta<1$ if $0 \leq \varrho<1$. Also note that the restriction (i) is precisely Assumption 4 in Morand \& Reffett (2007). This shows that the ME in their model is in fact unique whenever this restriction is imposed. Thus, their findings can considerably be strengthened if the additional properties of the operator $A$ identified above are exploited.

Condition (ii) in Theorem 1 is the natural extension of the uniqueness condition in Wang (1993) to the present more general setting. For the case with a classical production function $f$, it holds, e.g., if $f$ is of the CES form $f(k, \varepsilon)=\varepsilon g(k)$ where $g(k)=\varepsilon\left[1-\alpha+\alpha k^{\varrho}\right]^{\frac{1}{\varrho}}$, $0<\alpha<1$ and $0 \leq \varrho<1$ where $\varrho=0$ gives a Cobb-Douglas technology. The additional restriction $U_{12} \geq 0$ is imposed throughout in Morand \& Reffett (2007).

Finally, under the additional differentiability condition, (iii) permits to relax (ii) (which would imply $\frac{\mathcal{R}_{1}(k, \varepsilon) k}{\mathcal{R}(k, \varepsilon)} \geq-1$ ) while imposing an additional restriction on $U$. The latter generalizes (i) to the non-additive case and holds, e.g., for Cobb-Douglas utility $U\left(c^{y}, c^{o}\right)=\left(c^{y}\right)^{\alpha}\left(c^{o}\right)^{\beta}, \alpha, \beta>0$. Furthermore, for the classical CES production function mentioned above, $\frac{\mathcal{R}_{1}(k, \varepsilon) k}{\mathcal{R}(k, \varepsilon)}=\frac{k g^{\prime \prime}(k)}{g^{\prime}(k)}=-(1-\varrho) \frac{1-\alpha}{1-\alpha+\alpha k^{\varrho}}$ which implies that the restriction on $\mathcal{R}$ in (iii) holds iff $\varrho \geq-3$. The latter could further be relaxed if an upper bound on $k$ such as $k<f\left(k, \varepsilon^{\max }\right)$ - which is done in Morand \& Reffett (2007) - is imposed.

As a general insight, Theorem 1 shows that restrictions either on the consumption side (condition (i)) or the production side (condition (ii)) alone are already sufficient to induce a unique ME once it exists. It also reveals that the elasticities of the capital return function $\mathcal{R}$ and second-period marginal utility are key to the uniqueness of equilibrium while neither the wage function $\mathcal{W}$ nor the marginal utility of first-period consumption nor the transition $Q$ play a crucial role. As a consequence, multiple ME can occur only if capital income decreases very rapidly and the marginal utility of second-period consumption is very elastic. Thus, it seems rather difficult to obtain cases in which multiple MEs exist, and one may conclude that in the present class of models, uniqueness 
of $\mathrm{ME}$ - in fact, of equilibrium, as we show below in Theorem 3 - is a generic property. Also note that Theorem 1 does not ensure the existence of a ME. Imposing the additional restrictions of Morand \& Reffett (2007), existence follows directly from their results.

\section{Smoothness and monotonicity of $M E$}

In cases where the $\mathrm{ME}$ is unique, one may ask which additional properties of $\mathcal{K}$ can be inferred. In Wang (1993), the factor pricing functions $\mathcal{R}$ and $\mathcal{W}$ are both $C^{1}$ which implies that the map $\mathcal{K}$ is also $C^{1}$ and strictly increasing in his model. In the present case, a similar result holds in the sense that $\mathcal{K}$ essentially inherits the properties of the factor pricing functions. The result needs the following additional

\section{Assumption 4}

For all $\varepsilon \in \mathcal{E}$, the map $k \longmapsto \mathcal{R}(k, \varepsilon), k>0$ in (2b) satisfies the boundary behavior

$$
\lim _{k \rightarrow 0} \mathcal{R}(k, \varepsilon)=\infty \quad \text { and } \quad \lim _{k \rightarrow 0} k \mathcal{R}(k, \varepsilon)<\infty .
$$

Note that unlike Theorem 1 the following result also asserts the existence of a ME.

\section{Theorem 2}

Let Assumptions 1, 2, 3, and 4 and any of the hypotheses (i), (ii), or (iii) of Theorem 1 be satisfied. In addition, suppose $k \mapsto \mathcal{W}(k, \varepsilon)$ and $k \mapsto \mathcal{R}(k, \varepsilon)$ are continuous for all $\varepsilon \in \mathcal{E}$. Then the economy $\mathscr{E}$ has a unique $M E \mathcal{K} \in \mathscr{S}$ with the following properties:

(i) For all $\varepsilon \in \mathcal{E}, k \mapsto \mathcal{K}(k, \varepsilon), k>0$, is continuous. It is increasing if $U_{21} \geq 0$.

(ii) If $\mathcal{W}$ and $\mathcal{R}$ are continuous and $Q$ has the Feller property, then $\mathcal{K}$ is continuous.

(iii) If for all $\varepsilon \in \mathcal{E} k \mapsto \mathcal{W}(k, \varepsilon)$ and $k \mapsto \mathcal{R}(k, \varepsilon)$ are $C^{1}$, then $k \mapsto \mathcal{K}(k, \varepsilon)$ is $C^{1}$.

(iv) If, in addition to (iii), shocks are i.i.d., $\mathcal{E}$ is an interval, and $\mathcal{W}$ is $C^{1}$, then $\mathcal{K}$ is $C^{1}$. Further, if $\mathcal{W}_{2}>0$, then $\mathcal{K}_{2}>0$.

\section{Uniqueness of $S E$}

The previous discussion revolved around whether Markov equilibria are unique. This raises the question of whether there are other equilibria, i.e., recursive equilibria on a larger state space or non-recursive SE. A striking feature of the equilibrium structure from Section 2 is that the savings function $\mathcal{S}$ is independent of how the forecast $k_{t+1}$ was obtained. In other words, it is independent of any hypothesized law of motion and just depends on the value predicted for the following period. As a consequence, any equilibrium process - recursive or not - must satisfy the condition $k_{t+1} \in \Psi\left(k_{t}, \varepsilon_{t}\right)$ for all $t \geq 0$. This shows that if the ME is unique, i.e., the function $\Phi(x, \cdot)$ has a unique zero for all $x \in \mathbb{X}$, each state $x_{t}$ has a unique continuation value $k_{t+1}$ which is precisely the value determined by the ME. Thus, the equilibrium capital process $\left\{k_{t+1}\right\}_{t \geq 0}$ is uniquely defined. As the other equilibrium variables follow directly from this process, the equilibrium is in fact unique whenever the ME is unique. We state these insights as the following final theorem. The proof follows directly from the previous arguments. 


\section{Theorem 3}

Suppose the economy $\mathscr{E}$ has a unique ME. Then, this is also the unique SE of $\mathscr{E}$.

Note, however, that this result does not say that the number of ME is identical to the number of SE for the economy. The reason is that if there are multiple solutions to (7), then so-called Generalized Markov Equilibria (GME) exist which are obtained by randomizing over possible continuation values. This is the case studied in Wang (1994) using the powerful techniques of Duffie et al. (1994). Clearly, in this case, an uncountable number of distinct equilibrium processes can be generated.

\section{Conclusions}

Our analysis provides sufficient conditions under which a broad class of OLG economies with stochastic non-classical production and two-period lived consumers has at most one ME. The conditions obtained are quite general and should be easy to verify in applied work as they are stated directly on the primitives of the model.

One aspect not discussed in this paper is whether the ME gives rise to a Stationary Markov Equilibrium (SME) corresponding to an invariant distribution on the state space. Using different techniques, this issue is studied at length in Wang (1994) and Morand \& Reffett (2007). As the restrictions imposed in this paper are weaker than those in Morand \& Reffett (2007), their findings remain directly applicable in our setup if their additional restrictions are imposed.

As shown in Section 2 - and also in Wang (1994) - the key object for studying equilibria -recursive or not - is the equilibrium correspondence defined in (8). If this correspondence is multi-valued, i.e., not a function, the structure developed in Section 2 can be used to explicitly construct Generalized Markov equilibria by randomizing over different continuation values. While in this case, an uncountable number of SE exist, one could still quantify the equilibrium set by establishing bounds on the number of continuation values, i.e, the maximum the number of zeroes of the map $\Phi(x, \cdot)$ defined in $(6)$ for all choices $x \in \mathbb{X}$. For the latter purpose, the semi-algebraic approach developed in Kuebler \& Schmedders (2007) - although currently designed for static exchange economies - could become a useful tool. This might be an interesting avenue of future research.

\section{A Mathematical Appendix}

\section{A.1 Auxiliary results}

We begin by recalling some concepts and introducing some technical results that will be used subsequently. Given some topological space $\mathbb{X}$ with topology $\mathcal{T}_{\mathbb{X}} \subset \mathscr{P}(\mathbb{X})$, we 
endow subsets $\mathbb{Y}$ of $\mathbb{X}$ with the relative (trace) topology $\mathcal{T}_{\mathbb{Y}}:=\left\{\mathbb{O} \cap \mathbb{Y} \mid \mathbb{O} \in \mathcal{T}_{\mathbb{X}}\right\}$. Recall that if $\mathbb{Y}$ is open in $\mathbb{X}$, then $\mathcal{T}_{\mathbb{Y}} \subset \mathcal{T}_{\mathbb{X}}$. The Euclidean space $\mathbb{R}^{m}, m \geq 1$ is endowed with the usual topology which is generated by the Euclidean metric. Unless stated otherwise, continuity of functions is always understood with respect to these topologies.

A topological space $\mathbb{X}$ becomes a measurable space when endowed with the Borel $\sigma$ algebra $\mathscr{B}(\mathbb{X})$ which is generated by the open sets $\mathcal{T}_{\mathbb{X}}$. On subspaces $\mathbb{Y} \subset \mathbb{X}$ the Borel- $\sigma$ algebra $\mathscr{B}(\mathbb{Y})$ is generated by the relative topology $\mathcal{T}_{\mathbb{Y}}$ but also coincides with the trace $\sigma$-algebra, i.e., $\mathscr{B}(\mathbb{Y})=\{A \cap \mathbb{Y} \mid A \in \mathscr{B}(\mathbb{X})\}$, cf. Aliprantis \& Border (2007, p.138, Lemma 4.20). In particular, if $\mathbb{Y} \in \mathscr{B}(\mathbb{X})$, i.e., is measurable in $\mathbb{X}$, then $\mathscr{B}(\mathbb{Y}) \subset \mathscr{B}(\mathbb{X})$. Also recall that $A \in \mathscr{B}(X), B \in \mathscr{B}(Y)$ implies $A \times B \in \mathscr{B}(X \times Y)$.

The following lemmas will be used in the proofs of our results below.

\section{Lemma A.1 (Measurable Graph Theorem)}

A function $f: \mathbb{X} \longrightarrow \mathbb{Y}$ where both $\mathbb{X}$ and $\mathbb{Y}$ are complete separable metric spaces, is Borel-measurable, if and only if $\operatorname{graph}(f):=\{(x, y) \in \mathbb{X} \times \mathbb{Y} \mid y=f(x)\}$ is a Borelmeasurable subset of the product space $\mathbb{X} \times \mathbb{Y}$, i.e., $\operatorname{graph}(f) \in \mathscr{B}(\mathbb{X} \times \mathbb{Y})$.

Proof: See Buckley (1974, §3, Propositions 1 and 6).

\section{Lemma A.2 (Closed Graph Theorem)}

A function $f: \mathbb{X} \longrightarrow \mathbb{Y}$ from a topological space $\mathbb{X}$ into a compact Hausdorff space $\mathbb{Y}$ is continuous if and only if $\operatorname{graph}(f):=\{(x, y) \in \mathbb{X} \times \mathbb{Y} \mid y=f(x)\}$ is a closed subset of the product space $\mathbb{X} \times \mathbb{Y}$ (endowed with the product topology).

Proof: See Aliprantis \& Border (2007, p.51, Theorem 2.58).

\section{Lemma A.3}

Let $f: \mathbb{X} \longrightarrow \mathbb{Y}$ be a map between two topological spaces $\mathbb{X}$ (with topology $\mathcal{T}_{\mathbb{X}}$ ) and $\mathbb{Y}$. Suppose there is a sequence $\left\{\mathbb{X}_{n}\right\}_{n \geq 1}$ of open subsets $\left(\mathbb{X}_{n} \in \mathcal{T}_{\mathbb{X}}\right)$ of $\mathbb{X}$ which is increasing $\left(\mathbb{X}_{n} \subset \mathbb{X}_{n+1}\right)$ and converges to $\mathbb{X}$, i.e., $\mathbb{X}=\bigcup_{n \geq 1} \mathbb{X}_{n}$. Further, suppose that for each $n \geq 1$ the restricted map $f_{n}:=f_{\mid \mathbb{X}_{n}}: \mathbb{X}_{n} \longrightarrow \mathbb{Y}$ is continuous. Then $f$ is continuous.

Proof: Endow each $\mathbb{X}_{n}$ with the relative topology $\mathcal{T}_{n}:=\left\{\mathbb{U} \cap \mathbb{X}_{n} \mid \mathbb{U} \in \mathcal{T}_{\mathbb{X}}\right\}$. As $\mathbb{X}_{n}$ is open, $\mathcal{T}_{n} \subset \mathcal{T}_{\mathbb{X}}$ for all $n \geq 1$. Let $\mathbb{O}$ be an open subset of $\mathbb{Y}$. We have to show that $f^{-1}(\mathbb{O}) \in \mathcal{T}_{\mathbb{X}}$. As each $f_{n}$ is continuous, $f_{n}^{-1}(\mathbb{O}) \in \mathcal{T}_{n} \subset \mathcal{T}_{\mathbb{X}}$ and, therefore, $f_{n}^{-1}(\mathbb{O}) \in \mathcal{T}_{\mathbb{X}}$ for all $n \geq 1$. We claim that $f^{-1}(\mathbb{O})=\bigcup_{n \geq 1} f_{n}^{-1}(\mathbb{O})$. Let $x \in f^{-1}(\mathbb{O})$ be arbitrary. Since $\mathbb{X}=\bigcup_{n>1} \mathbb{X}_{n}$, there is some $\mathbb{X}_{m}$ such that $x \in \mathbb{X}_{m}$ and $f(x)=f_{m}(x) \in \mathbb{O}$. Hence, $x \in$ $f_{m}^{-1}(\mathbb{O}) \subset \bigcup_{n \geq 1} f_{n}^{-1}(\mathbb{O})$ which, since $x$ was arbitrary shows that $f^{-1}(\mathbb{O}) \subset \bigcup_{n \geq 1} f_{n}^{-1}(\mathbb{O})$. Conversely, let $x \in \bigcup_{n \geq 1} f_{n}^{-1}(\mathbb{O})$. Then, $x \in f_{m}^{-1}(\mathbb{O})$ for some $m$, i.e., $x \in \mathbb{X}_{m}^{-} \subset \mathbb{X}$ and $f_{m}(x)=f(x) \in \mathbb{O}$. Conclude that $x \in f^{-1}(\mathbb{O})$ which, since $x$ was arbitrary, implies $f^{-1}(\mathbb{O}) \supset \bigcup_{n \geq 1} f_{n}^{-1}(\mathbb{O})$. Thus, we have shown that $f^{-1}(\mathbb{O})$ is the union of open sets $f_{n}^{-1}(\mathbb{O}) \in \mathcal{T}_{\mathbb{X}}$. Since any union of open sets is open again, this implies $f^{-1}(\mathbb{O}) \in \mathcal{T}_{\mathbb{X}}$. 


\section{Lemma A.4}

Let $f: \mathbb{X} \longrightarrow \mathbb{Y}$ be a map between two topological spaces $\mathbb{X}$ and $\mathbb{Y}$ endowed with the Borel $\sigma$-algebras $\mathscr{B}(\mathbb{X})$ and $\mathscr{B}(\mathbb{Y})$. Suppose there is a sequence $\left\{\mathbb{X}_{n}\right\}_{n \geq 1}$ of measurable subsets $\left(\mathbb{X}_{n} \in \mathscr{B}(\mathbb{X})\right.$ ) of $\mathbb{X}$ which is increasing $\left(\mathbb{X}_{n} \subset \mathbb{X}_{n+1}\right)$ and converges to $\mathbb{X}$, i.e., $\mathbb{X}=\bigcup_{n \geq 1} \mathbb{X}_{n}$. Further, suppose that for each $n \geq 1$ the restricted map $f_{n}:=f_{\mid \mathbb{X}_{n}}$ : $\mathbb{X}_{n} \longrightarrow \mathbb{Y}$ is measurable. Then $f$ is measurable.

Proof: Endow each $\mathbb{X}_{n}$ with the trace- $\sigma$ algebra $\mathscr{B}_{n}:=\left\{A \cap \mathbb{X}_{n} \mid A \in \mathscr{B}(\mathbb{X})\right\}$. As $\mathbb{X}_{n}$ is measurable, $\mathscr{B}_{n} \subset \mathscr{B}(\mathbb{X})$ for $n \geq 1$. Let $A \in \mathscr{B}(\mathbb{Y})$. We show that $f^{-1}(A) \in \mathscr{B}(\mathbb{X})$. As each $f_{n}$ is $\mathscr{B}_{n}-\mathscr{B}(\mathbb{Y})$ measurable, $f_{n}^{-1}(A) \in \mathscr{B}_{n} \subset \mathscr{B}(\mathbb{X})$ and, therefore, $f_{n}^{-1}(A) \in \mathscr{B}(\mathbb{X})$ for all $n \geq 1$. Following the exact same arguments as in the proof of Lemma A.3 (with 'open' replaced by 'measurable'), it is straightforward to show that $f^{-1}(A)=$ $\bigcup_{n \geq 1} f_{n}^{-1}(A)$, i.e., $f^{-1}(A)$ is the countable union of measurable subsets $f_{n}^{-1}(A) \in \mathscr{B}(\mathbb{X})$. As $\mathscr{B}(\mathbb{X})$ is closed under countable unions, this implies $f^{-1}(A) \in \mathscr{B}(\mathbb{X})$, as claimed.

\section{A.2 Proof of Lemma 2.1}

Define $\mathbb{U}:=\left\{(w, s) \in \mathbb{R}_{++}^{2} \mid s<w\right\}, \mathbb{V}:=\mathbb{R}_{++} \times \mathcal{E} \times \mathbb{U}$, and let $v: \mathbb{R}_{++} \times \mathbb{U} \longrightarrow \mathbb{R}$,

$$
v(r, w, s):=-U_{1}(w-s, s r)+r U_{2}(w-s, s r) .
$$

The map $v$ is $C^{1}$ and, therefore, measurable. As the composition of measurable functions is measurable, Assumption 2 implies measurability of $\left(k^{\prime}, \varepsilon^{\prime}, w, s\right) \mapsto v\left(\mathcal{R}\left(k^{\prime}, \varepsilon^{\prime}\right), w, s\right)$ and, invoking Assumption 3, of the function $V: \mathbb{V} \longrightarrow \mathbb{R}$,

$$
V\left(k^{\prime}, \varepsilon, w, s\right):=\int_{\mathcal{E}} v\left(\mathcal{R}\left(k^{\prime}, \varepsilon^{\prime}\right), w, s\right) Q\left(\varepsilon, d \varepsilon^{\prime}\right) .
$$

Using the same arguments as in Section 2, the function $\left.V\left(k^{\prime}, \varepsilon, w, \cdot\right):\right] 0, w[\longrightarrow \mathbb{R}$ has a unique zero $0<s<w$ for each $\left(k^{\prime}, \varepsilon, w\right) \in \mathbb{S}:=\mathbb{R}_{++} \times \mathcal{E} \times \mathbb{R}_{++}$which may be written as a function $\tilde{S}: \mathbb{S} \longrightarrow \mathbb{R}_{+}, s=\tilde{\mathcal{S}}\left(k^{\prime}, \varepsilon, w\right)$. We will show that $\tilde{S}$ is measurable. This and measurability of $\mathcal{W}$ due to Assumption 2 will then imply that $\mathcal{S}\left(k, \varepsilon, k^{\prime}\right)=$ $\tilde{\mathcal{S}}\left(k^{\prime}, \varepsilon, \mathcal{W}(k, \varepsilon)\right)$ is measurable. ${ }^{4}$

For $n \geq 1$, define $\mathbb{S}_{n}:=\left[\frac{1}{n}, n\right] \times \mathcal{E} \times\left[\frac{1}{n}, n\right] \subset \mathbb{S}$. Observe that $\left\{\mathbb{S}_{n}\right\}_{n \geq 1}$ is an increasing $\left(\mathbb{S}_{n} \subset \mathbb{S}_{n+1}\right)$ sequence of measurable $\left(\mathbb{S}_{n} \in \mathscr{B}(\mathbb{S})\right)$ subsets of $\mathbb{S}$ that converges to $\mathbb{S}=$ $\bigcup_{n \geq 1} \mathbb{S}_{n}$. Thus, by Lemma A.4, it suffices to show that each $\tilde{\mathcal{S}}_{n}:=\tilde{\mathcal{S}}_{\mathbb{S}_{n}}: \mathbb{S}_{n} \longrightarrow[0, n]$

\footnotetext{
${ }^{4}$ Until here, the argument is similar to the proof of Lemma 5 in Morand \& Reffett (2007). At this point, however, their argument concludes that continuity of $\tilde{\mathcal{S}}(\cdot, \varepsilon)$ in $w$ and $k^{\prime}$ for each fixed $\varepsilon \in \mathcal{E}$ implies measurability of $\mathcal{S}$. While this is correct if shocks are i.i.d., it neglects that, in general, there will also be a direct influence of $\varepsilon$ on $\tilde{\mathcal{S}}$ through the transition $Q$. Then, the previous argument seems no longer valid to infer measurability of $\tilde{\mathcal{S}}$. The following proof presents an alternative which fixes this problem and also shows that measurability of $\tilde{\mathcal{S}}$ obtains even under the more general restrictions of this paper.
} 
is measurable. Observe that both the domain and range of $\tilde{\mathcal{S}}_{n}$ being closed subsets of complete separable metric spaces are complete separable metric spaces. Thus, by Lemma A.2, $\tilde{\mathcal{S}}_{n}$ is measurable if and only if $\operatorname{graph}\left(\tilde{\mathcal{S}}_{n}\right)$ is a measurable subset of $\mathbb{S}_{n} \times[0, n]$, i.e., $\operatorname{graph}\left(\tilde{\mathcal{S}}_{n}\right) \in \mathscr{B}\left(\mathbb{S}_{n} \times[0, n]\right)$.

For $n \geq 1$, define $\mathbb{V}_{n}:=\left\{\left(k^{\prime}, \varepsilon, w, s\right) \in\left[\frac{1}{n}, n\right] \times \mathcal{E} \times\left[\frac{1}{n}, n\right] \times[0, n] \mid s<w\right\}=\mathbb{V} \cap\left(\mathbb{S}_{n} \times[0, n]\right)$ which is a measurable subset of $\mathbb{V}$. Consider the restriction $V_{n}:=V_{\mathbb{V}_{n}}: \mathbb{V}_{n} \longrightarrow \mathbb{R}$ which is measurable as the restriction of a measurable map $V$ to a measurable subset of its domain. As $\{0\}$ is a measurable subset of $\mathbb{R}$ this implies that $V_{n}^{-1}(\{0\})=\left\{\left(k^{\prime}, \varepsilon, w, s\right) \in\right.$ $\left.\mathbb{V}_{n} \mid V_{n}\left(k^{\prime}, \varepsilon, w, s\right)=0\right\}=\left\{\left(k^{\prime}, \varepsilon, w, s\right) \in \mathbb{S}_{n} \times[0, n] \mid s=\tilde{\mathcal{S}}_{n}\left(k^{\prime}, \varepsilon, w\right)\right\}=\operatorname{graph}\left(\tilde{\mathcal{S}}_{n}\right)$ is a measurable subset of $\mathbb{V}_{n}$, i.e., graph $\left(\tilde{\mathcal{S}}_{n}\right) \in \mathscr{B}\left(\mathbb{V}_{n}\right)$. Since $\mathbb{V}_{n}$ is a measurable subset of $\mathbb{S}_{n} \times[0, n], \mathscr{B}\left(\mathbb{V}_{n}\right) \subset \mathscr{B}\left(\mathbb{S}_{n} \times[0, n]\right)$ which implies that $\operatorname{graph}\left(\tilde{\mathcal{S}}_{n}\right) \in \mathscr{B}\left(\mathbb{S}_{n} \times[0, n]\right)$

\section{A.3 Proof of Lemma 2.3}

(i) Suppose the economy has a unique $\mathrm{ME} \mathcal{K}$. Then, $\mathcal{K}$ is a measurable selection of the correspondence $\Psi$ defined in (8). By contradiction, suppose for some $\tilde{x} \in \mathbb{X}$, the function $\Phi(\tilde{x}, \cdot)$ has at least two zeroes, say $k_{1}$ and $k_{2}$. W.l.o.g., suppose $k_{1}=\mathcal{K}(\tilde{x})$. Then, the function $\mathcal{K}_{2}: \mathbb{X} \longrightarrow \mathbb{R}_{++}$,

$$
\mathcal{K}_{2}(x):=\mathcal{K}(x)+1_{\{\tilde{x}\}}(x)\left(k_{2}-k_{1}\right)= \begin{cases}k_{2} & x=\tilde{x} \\ \mathcal{K}(x) & \text { otherwise }\end{cases}
$$

is a measurable function that satisfies $\mathcal{K}_{2}(x) \in \Psi(x)$ for all $x \in \mathbb{X}$, i.e., is another ME, contradicting uniqueness of the ME.

Conversely, suppose for all $\tilde{x} \in \mathbb{X}$, the function $\Phi(\tilde{x}, \cdot)$ has a unique zero. In this case, the correspondence $\Psi: \mathbb{X} \longrightarrow \mathbb{R}_{++}$is a function whose domain $\mathbb{X}=\mathbb{R}_{++}^{2} \times \mathcal{E}$ is the product of an open and a closed subset of $\mathbb{R}$ which is a Polish space. As open and closed subsets of Polish spaces are Polish and so are their product, both the domain and range of $\Psi$ are Polish spaces. By Aliprantis \& Border (2007, Theorem 12.28, p.450), $\Psi$ is measurable if and only if $\operatorname{graph}(\Psi)$ is a measurable subset of $\mathbb{X} \times \mathbb{R}_{++}$. By assumption, $\Phi: \mathbb{X} \times \mathbb{R}_{++} \longrightarrow \mathbb{R}$ is a measurable function which implies that $\Phi^{-1}(\{0\})=\left\{\left(x, k^{\prime}\right) \in\right.$ $\left.\mathbb{X} \times \mathbb{R}_{++} \mid \Phi\left(x, k^{\prime}\right)=0\right\}=\left\{\left(x, k^{\prime}\right) \in \mathbb{X} \times \mathbb{R}_{++} \mid k^{\prime}=\Psi(x)\right\}=\operatorname{graph}(\Psi)$ is a measurable subset of $\mathbb{X} \times \mathbb{R}_{++}$.

(ii) If for some $\tilde{x} \in \mathbb{X}, \Phi(\tilde{x}, \cdot)$ fails to have a zero, then $\Psi(\tilde{x})=\emptyset$. As any ME is a measurable selection of $\Psi$, there will be no ME in this case. If, for all $\tilde{x} \in \mathbb{X}, \Phi(\tilde{x}, \cdot)$ has precisely one zero, then a ME exists by (i).

\section{A.4 Proof of Theorem 1}

Let $x=(k, \varepsilon) \in \mathbb{X}$ and $w:=\mathcal{W}(k, \varepsilon)>0$ be arbitrary but fixed. Using Lemma 2.3(ii), we show that the map $\Phi(x, \cdot): \mathbb{R}_{++} \longrightarrow \mathbb{R}$ defined in (6) has at most one zero $k^{\prime}>0$. 
Define $\tilde{\mathcal{S}}$ as in the proof of Lemma 2.1. Then, using (6) and (A.2)

$$
\Phi\left(x, k^{\prime}\right)=0 \quad \Leftrightarrow \quad k^{\prime}=\tilde{\mathcal{S}}\left(k^{\prime}, \varepsilon, w\right) \quad \Leftrightarrow \quad V\left(k^{\prime}, w, \varepsilon, k^{\prime}\right)=0 .
$$

Thus, letting $G\left(w, \varepsilon, k^{\prime}\right):=V\left(k^{\prime}, w, \varepsilon, k^{\prime}\right)$ with $V$ defined as in (A.2), it suffices to show that $G(w, \varepsilon, \cdot):] 0, w[\longrightarrow \mathbb{R}$ is strictly monotonic. Defining

$$
g\left(w, k^{\prime}, \varepsilon^{\prime}\right):=-U_{1}\left(w-k^{\prime}, k^{\prime} \mathcal{R}\left(k^{\prime}, \varepsilon^{\prime}\right)\right)+\mathcal{R}\left(k^{\prime}, \varepsilon^{\prime}\right) U_{2}\left(w-k^{\prime}, k^{\prime} \mathcal{R}\left(k^{\prime}, \varepsilon^{\prime}\right)\right)
$$

the function $G$ may be written as

$$
G\left(w, \varepsilon, k^{\prime}\right)=\int_{\mathcal{E}} g\left(w, k^{\prime}, \varepsilon^{\prime}\right) Q\left(\varepsilon, d \varepsilon^{\prime}\right) .
$$

As integration preserves monotonicity, it suffices to show that $k^{\prime} \mapsto g\left(w, k^{\prime}, \varepsilon^{\prime}\right)$ is strictly monotonic - in fact, decreasing - in each of the three cases below.

(i) Under the hypotheses, the map $g$ in (A.4) takes the form

$$
g\left(w, k^{\prime}, \varepsilon^{\prime}\right)=-u^{\prime}\left(w-k^{\prime}\right)+f\left(k^{\prime}, \mathcal{R}\left(k^{\prime}, \varepsilon^{\prime}\right)\right)
$$

where $f: \mathbb{R}_{++}^{2} \longrightarrow \mathbb{R}_{++}, f(x, y):=y v^{\prime}(y x)$. The first term in (A.6) is a strictly decreasing function of $k^{\prime}$. Thus, it suffices to show that $k^{\prime} \mapsto f\left(k^{\prime}, \mathcal{R}\left(k^{\prime}, \varepsilon^{\prime}\right)\right)$ is decreasing for all $\varepsilon^{\prime} \in \mathcal{E}$, which follows directly from $f_{1}(x, y)=y^{2} v^{\prime \prime}(y x)<0 \leq f_{2}(x, y)=v^{\prime}(y x)+$ $y x v^{\prime \prime}(y x)$ and $k^{\prime} \mapsto \mathcal{R}\left(k^{\prime}, \varepsilon^{\prime}\right)$ being strictly decreasing by Assumption 2 .

(ii) Under the hypotheses, it is straightforward to show that $k^{\prime} \mapsto g\left(w, k^{\prime}, \varepsilon^{\prime}\right), 0<k^{\prime}<w$ defined in (A.4) is strictly decreasing for all $\left(w, \varepsilon^{\prime}\right) \in \mathbb{R}_{++} \times \mathcal{E}$.

(iii) Under the additional hypothesis, the function $k^{\prime} \mapsto g\left(w, \varepsilon^{\prime}, k^{\prime}\right)$ defined in (A.4) is continuously differentiable for all $\left(w, \varepsilon^{\prime}\right) \in \mathbb{R}_{++} \times \mathcal{E}$ and so is $G$. As differentiation and integration over a compact set may be interchanged, the partial derivative of $G$ computes

$$
G_{3}\left(w, \varepsilon, k^{\prime}\right)=\int_{\mathcal{E}} g_{3}\left(w, k^{\prime}, \varepsilon^{\prime}\right) Q\left(\varepsilon, d \varepsilon^{\prime}\right) .
$$

It suffices to show that $g_{3}<0$. Dropping the respective arguments for convenience, the derivative of (A.4) may be written as

$$
g_{3}\left(w, \varepsilon^{\prime}, k^{\prime}\right)=\mathcal{R}_{1}\left[U_{2}+k^{\prime} \mathcal{R} U_{22}\right]+U_{11}-2 \mathcal{R} U_{21}+\mathcal{R}^{2} U_{22}-U_{21} k^{\prime} \mathcal{R}_{1} .
$$

The first term is strictly negative as $\mathcal{R}_{1}<0$ and the bracketed term is strictly positive by assumption. Suppose first that $U_{21} \leq 0$. Then, the last term in (A.8) is negative as well, so we need to show that $U_{11}-2 \mathcal{R} U_{21}+\mathcal{R}^{2} U_{22} \leq 0$ or, using $U_{i i}<0$ for $i \in\{1,2\}$

$$
M:=\left|U_{11}\right|-2 \mathcal{R}\left|U_{21}\right|+\mathcal{R}^{2}\left|U_{22}\right| \geq 0
$$

The concavity of $U$ implies a negative semi-definite Hessian matrix, so $U_{11} U_{22} \geq U_{12}^{2}$, which may be restated as $\left|U_{12}\right| \leq\left|U_{11}\right|^{\frac{1}{2}}\left|U_{22}\right|^{\frac{1}{2}}$. Substituting this result into (A.9) gives

$$
M \geq\left|U_{11}\right|-2 \mathcal{R}\left|U_{11}\right|^{\frac{1}{2}}\left|U_{22}\right|^{\frac{1}{2}}+\mathcal{R}^{2}\left|U_{22}\right|=\left(\left|U_{11}\right|^{\frac{1}{2}}-\mathcal{R}\left|U_{22}\right|^{\frac{1}{2}}\right)^{2} \geq 0 .
$$


Second, suppose that $k^{\prime} \mathcal{R}_{1} \geq-4 \mathcal{R}$. If $U_{21}\left(w-k^{\prime}, k^{\prime} \mathcal{R}\left(k^{\prime}, \varepsilon^{\prime}\right)\right) \leq 0$, the argument of the previous step remains unchanged, so suppose $U_{21}\left(w-k^{\prime}, k^{\prime} \mathcal{R}\left(k^{\prime}, \varepsilon^{\prime}\right)\right)>0$. Then,

$$
U_{11}-2 \mathcal{R} U_{21}+\mathcal{R}^{2} U_{22}-U_{21} k \mathcal{R}_{1} \leq U_{11}+2 \mathcal{R} U_{21}+\mathcal{R}^{2} U_{22}=-M
$$

with $M$ defined in (A.9). As shown before, $M \geq 0$, which proves the claim.

\section{A.5 Proof of Theorem 2}

Define the maps $g$ and $G$ as in (A.4) and (A.5). Note that the continuity assumption implies that $g$ is a continuous function in $k^{\prime}$. As integration over a compact set preserves continuity, $G$ is continuous in $k^{\prime}$ for all $(w, \varepsilon) \in \mathbb{R}_{++} \times \mathcal{E}$. The existence of a ME will follow from Lemma 2.3 (i) if we show that $G(w, \varepsilon, \cdot)$ has a unique zero for all $(w, \varepsilon) \in \mathbb{R}_{++} \times \mathcal{E}$. To this end, observe that Assumption 4 implies the boundary behavior $\lim _{k \searrow w} g\left(w, \varepsilon^{\prime}, k\right)=-\infty$ and $\lim _{k \nearrow_{0}} g\left(w, \varepsilon^{\prime}, k\right)=\infty$. This being true for all $\varepsilon^{\prime} \in \mathcal{E}$ implies that $\lim _{k \searrow w} G(w, \varepsilon, k)=-\infty$ and $\lim _{k \nearrow_{0}} G(w, \varepsilon, k)=\infty$ for all $(w, \varepsilon) \in \mathbb{R}_{++} \times \mathcal{E}$. By continuity of $G(w, \varepsilon, \cdot)$, this ensures existence of a zero which is necessarily unique by monotonicity and defined by an implicit function $\tilde{\mathcal{K}}: \mathbb{R}_{++} \times \mathcal{E} \longrightarrow \mathbb{R}_{++}$. By Lemma 2.3 , the function $\mathcal{K}: \mathbb{X} \longrightarrow \mathbb{R}_{++}, \mathcal{K}(k, \varepsilon):=\tilde{\mathcal{K}}(\mathcal{W}(k, \varepsilon), \varepsilon)$ is the unique ME. Clearly, if $U_{12} \geq 0$, then $g$ and $G$ are both strictly increasing in $w$, which implies that $\tilde{\mathcal{K}}(\cdot, \varepsilon)$ is strictly increasing. It then follows from Assumption $4(\mathrm{ii})$ that $\mathcal{K}(\cdot, \varepsilon)$ is increasing.

(i) Let $\varepsilon \in \mathcal{E}$ be arbitrary but fixed. Define $\mathbb{U}:=\left\{\left(w, k^{\prime}\right) \in \mathbb{R}_{++}^{2} \mid 0<k^{\prime}<w\right\}$ and the map $G^{\varepsilon}: \mathbb{U} \longrightarrow \mathbb{R}, G^{\varepsilon}(w, k):=G(w, \varepsilon, k)$. Under the hypotheses, $G^{\varepsilon}$ is a continuous function as the integrand is continuous and integration over a compact set preserves continuity. As shown in the proof of Theorem 1, for each $w>0$ the map $\left.G^{\varepsilon}(w, \cdot):\right] 0, w[\longrightarrow \mathbb{R}$ is strictly decreasing and, therefore, has a unique zero determined by some map $\tilde{\mathcal{K}}^{\varepsilon}: \mathbb{R}_{++} \longrightarrow \mathbb{R}_{++}$. We show that $\tilde{\mathcal{K}}^{\varepsilon}$ is continuous.

For $n>1$, let $\delta_{n}>0$ be a small number such that $\delta_{n}<\frac{1}{2 n}$. Define the compact set $\mathbb{U}_{n}:=\left\{(w, k) \in \mathbb{R}_{++}^{2} \mid \frac{1}{n} \leq w \leq n, \delta_{n} \leq k \leq w-\delta_{n}\right\}$ and consider the restriction $G_{n}^{\varepsilon}:=G_{\mid \mathbb{U}_{n}}^{\varepsilon}: \mathbb{U}_{n} \longrightarrow \mathbb{R}$. Clearly, $G_{n}^{\varepsilon}$ is continuous as the restriction of a continuous function to a subset of its domain. We seek to determine $\delta_{n}$ such that each $G_{n}^{\varepsilon}(w, \cdot)$ : $\left[\delta_{n}, w-\delta_{n}\right] \longrightarrow \mathbb{R}$ has a - necessarily unique - zero for all $w \in\left[\frac{1}{n}, n\right]$ determined by $\hat{\mathcal{K}}_{n}^{\varepsilon}:\left[\frac{1}{n}, n\right] \longrightarrow[0, n]$. Then, $\hat{\mathcal{K}}_{n}^{\varepsilon}$ will be the restriction $\tilde{\mathcal{K}}^{\varepsilon}$ to $\left[\frac{1}{n}, n\right]$. Recall that $G_{n}^{\varepsilon}(w, \cdot)$ is continuous and strictly decreasing for all $w \in\left[\frac{1}{n}, n\right]$. Thus, to ensure that $\hat{\mathcal{K}}_{n}^{\varepsilon}$ is welldefined, it suffices to have $G_{n}^{\varepsilon}\left(w, \delta_{n}\right)<0<G_{n}^{\varepsilon}\left(w, w-\delta_{n}\right)$ for all $w \in\left[\frac{1}{n}, n\right]$. For $\delta_{n}>0$, define

$$
\begin{aligned}
G_{\max }^{\varepsilon}\left(\delta_{n}\right) & :=\max _{w}\left\{G^{\varepsilon}\left(w, w-\delta_{n}\right) \mid w \in\left[\frac{1}{n}, n\right]\right\} \\
G_{\min }^{\varepsilon}\left(\delta_{n}\right) & :=\min _{w}\left\{G^{\varepsilon}\left(w, \delta_{n}\right) \mid w \in\left[\frac{1}{n}, n\right]\right\}
\end{aligned}
$$


which are well-defined due to continuity of $G^{\varepsilon}$ and compactness of $\left[\frac{1}{n}, n\right]$. Note that $G_{\max }^{\varepsilon}$ is strictly increasing in $\delta_{n}$ and $\lim _{\delta \rightarrow 0} G^{\varepsilon}(w, w-\delta)=-\infty$ for all $w>0$ implies $G_{\max }^{\varepsilon}\left(\delta_{n}\right)<0$ for $\delta_{n}$ sufficiently small. Likewise, $G_{\min }^{\varepsilon}$ is strictly decreasing in $\delta_{n}$ and $\lim _{\delta \rightarrow 0} G^{\varepsilon}(w, \delta)=\infty$ for all $w>0$, implies $G_{\min }^{\varepsilon}\left(\delta_{n}\right)>0$ for $\delta_{n}$ sufficiently small. Thus, choosing $\delta_{n}$ small enough such that $G_{\max }^{\varepsilon}\left(\delta_{n}\right)<0<G_{\min }^{\varepsilon}\left(\delta_{n}\right)$ implies $G^{\varepsilon}\left(w, w-\delta_{n}\right)<$ $0<G^{\varepsilon}\left(w, \delta_{n}\right)$ for all $w \in\left[\frac{1}{n}, n\right]$. Then, for each $w \in\left[\frac{1}{n}, n\right]$ there exists a unique zero $k_{0} \in\left[\delta_{n}, w-\delta_{n}\right]$ of $G_{n}^{\varepsilon}(w, \cdot)$ determined by the function $\hat{\mathcal{K}}_{n}^{\varepsilon}:\left[\frac{1}{n}, n\right] \longrightarrow[0, n]$.

We show that each $\hat{\mathcal{K}}_{n}^{\varepsilon}$ is continuous. Employing Lemma A.2, it suffices to show that $\operatorname{graph}\left(\hat{\mathcal{K}}_{n}^{\varepsilon}\right)$ is a closed subset of $\left[\frac{1}{n}, n\right] \times[0, n]$. As $\{0\}$ is a closed subset of $\mathbb{R}$, continuity of $G_{n}^{\varepsilon}$ implies that $\left(G_{n}^{\varepsilon}\right)^{-1}(\{0\})$ is a closed subset of $\mathbb{U}_{n}$. But $\left(G_{n}^{\varepsilon}\right)^{-1}(\{0\})=\{(w, k) \in$ $\left.\mathbb{U}_{n} \mid G_{n}^{\varepsilon}(w, k)=0\right\}=\left\{(w, k) \in \mathbb{U}_{n} \mid k=\hat{\mathcal{K}}_{n}^{\varepsilon}(w)\right\}=\operatorname{graph}\left(\hat{\mathcal{K}}_{n}^{\varepsilon}\right) . \operatorname{Thus}, \operatorname{graph}\left(\hat{\mathcal{K}}_{n}^{\varepsilon}\right)$ is a closed subset of $\mathbb{U}_{n}$. As $\mathbb{U}_{n}$ is a closed subset of $\left[\frac{1}{n}, n\right] \times[0, n], \operatorname{graph}\left(\hat{\mathcal{K}}_{n}^{\varepsilon}\right)$ is also closed in $\left[\frac{1}{n}, n\right] \times[0, n]$.

Now let $\tilde{\mathcal{K}}_{n}^{\varepsilon}$ be the restriction of $\hat{\mathcal{K}}_{n}^{\varepsilon}$ to the open subset $\left.\mathbb{S}_{n}:=\right] \frac{1}{n}, n\left[\right.$. Clearly, each $\tilde{\mathcal{K}}_{n}^{\varepsilon}$ is continuous as the restriction of a continuous map to a subset of its domain. Moreover, $\tilde{\mathcal{K}}_{n}^{\varepsilon}$ is also the restriction of $\tilde{\mathcal{K}}^{\varepsilon}$ to $\mathbb{S}_{n}$ and $\left\{\mathbb{S}_{n}\right\}_{n>1}$ is an increasing sequence of open subsets of $\mathbb{R}_{++}=\cup_{n>1} \mathbb{S}_{n}$. Thus, continuity of $\tilde{\mathcal{K}}^{\varepsilon}$ for all $\varepsilon \in \mathcal{E}$ follows from Lemma A.3. Since compositions of continuous functions are continuous, this implies continuity of $\mathcal{K}(\cdot, \varepsilon)$.

(ii) Under the additional hypotheses, the function $g$ in (A.4) is continuous. The Feller property of $Q$ then implies that $G$ in (A.5) is continuous as well. One can now slightly modify the arguments of the previous step to show that $\mathcal{K}$ is a continuous function.

(iii) Let $\varepsilon \in \mathcal{E}$ be arbitrary but fixed. Under the additional hypotheses, the function $G^{\varepsilon}$ defined in (i) is $C^{1}$. Repeating the proof of Theorem 1 under the additional differentiability condition, it is straightforward to show that $G_{2}^{\varepsilon}(w, k)>0$. Thus, an application of the implicit function theorem yields that $\tilde{\mathcal{K}}^{\varepsilon}$ defined in (i) is $C^{1}$ with derivative $\tilde{\mathcal{K}}_{1}^{\varepsilon}(w)=-G_{1}^{\varepsilon}\left(w, \mathcal{K}^{\varepsilon}(w)\right)\left[G_{2}^{\varepsilon}\left(w, \mathcal{K}^{\varepsilon}(w)\right)\right]^{-1}$.

(iv) If the shocks are i.i.d, the functions $G=G^{\varepsilon}$ and $\tilde{\mathcal{K}}=\tilde{\mathcal{K}}^{\varepsilon}$ defined in the previous steps are independent of $\varepsilon$. As shown in (iii), $\tilde{\mathcal{K}}$ is $C^{1}$ which implies that $\mathcal{K}=\tilde{\mathcal{K}} \circ \mathcal{W}$ is $C^{1}$ under the hypothesis. If, in addition, $\mathcal{W}_{2}>0$ then clearly $\mathcal{K}_{2}>0$.

\section{References}

Aliprantis, C. D. \& K. C. Border (2007): Infinite Dimensional Analysis. SpringerVerlag, Berlin a.o.

Buckley, J. (1974): "Graphs of Measurable Functions", Proceedings of the American Mathematical Society, 44, 78-80. 
Coleman, W. J. II (1991): "Equilibrium in a Production Economy with an Income Tax", Econometrica, 59, 1091-1104.

De la Croix, D. \& P. Michel (2002): A Theory of Economic Growth - Dynamics and Policy in Overlapping Generations. Cambridge University Press.

Duffie, D., J. Geanakoplos, A. Mas-Colell \& A. Mclennan (1994): "Stationary Markov Equilibria", Econometrica, 62(4), 745-781.

Feng, Z., J.Miao, A. Peralta-Alva \& M.Santos (2012): "Numerical Simulation of Nonoptimal Dynamic Equilibrium Models", Working paper, Federal Reserve Bank of St.Louis.

Greenwood, J. \& G. Huffman (1995): "On the Existence of Nonoptimal Equilibria in Dynamic Stochastic Economies", Journal of Economic Theory, 65, 611-623.

Kübler, F. \& H. Polemarchakis (2004): "Stationary Markov Equilibria for Overlapping Generations", Economic Theory, 24(3), 623-643.

Kuebler, F. \& K. Schmedders (2007): "Competitive Equilibria in Semi-Algebraic economies", Discussion paper.

Morand, O. F. \& K. L. Reffett (2007): "Stationary Markovian Equilibrium in Overlapping Generations Models with Stochastic Nonclassical Production and Markov Shocks", Journal of Mathematical Economics, 43, 501-522.

WANG, Y. (1993): "Stationary Equilibria in an Overlapping Generations Economy with Stochastic Production", Journal of Economic Theory, 61(2), 423-435.

Wang, Y. (1994): "Stationary Markov Equilibria in an OLG Model with Correlated Production Shocks", International Economic Review, 35(3), 731-744. 


\section{Working Paper Series in Economics}

recent issues

No. 46 Marten Hillebrand: Uniqueness of Markov equilibrium in stochastic OLG models with nonclassical production, November 2012

No. 45 Philipp Schuster and Marliese Uhrig-Homburg: The term structure of bond market liquidity conditional on the economic environment: an analysis of government guaranteed bonds, November 2012

No. 44 Young Shin Kim, Rosella Giacometti, Svetlozar T. Rachev, Frank J. Fabozzi, Domenico Mignacca: Measuring financial risk and portfolio optimization with a non-Gaussian multivariate model, August 2012

No. 43 Zuodong Lin, Svetlozar T. Rachev, Young Shin Kim, Frank J. Fabozzi: Option pricing with regime switching tempered stable processes, August 2012

No. 42 Siegfried K. Berninghaus, Werner Güth, Stephan Schosser: Backward induction or forward reasoning? An experiment of stochastic alternating offer bargaining, July 2012

No. 41 Siegfried Berninghaus, Werner Güth, King King Li: Approximate truth of perfectness - an experimental test, June 2012

No. 40 Marten Hillebrand and Tomoo Kikuchi: A mechanism for booms and busts in housing prices, May 2012

No. 39 Antje Schimke: Entrepreneurial aging and employment growth in the context of extreme growth events, May 2012

No. 38 Antje Schimke, Nina Teichert, Ingrid Ott: Impact of local knowledge endowment on employment growth in nanotechnology, February 2012

No. 37 Siegfried K. Berninghaus, Lora Todorova, Bodo Vogt: A simple questionnaire can change everything - are strategy choices in coordination games stable?, December 2011 